\title{
Improvement in hemodynamic performance, exercise capacity, inflammatory profile, and left ventricular reverse remodeling after intracoronary delivery of mesenchymal stem cells in an experimental model of pressure overload hypertrophy
}

\author{
Ezequiel J. Molina, MD, ${ }^{\text {a }}$ Jon Palma, MS, ${ }^{a}$ Dipin Gupta, MD, ${ }^{a}$ Denise Torres, MD, ${ }^{a}$ John P. Gaughan, PhD, ${ }^{b}$
} Steven Houser, $\mathrm{PhD}{ }^{\mathrm{b}}$ and Mahender Macha, $\mathrm{MD}^{\mathrm{c}}$

丹 Supplemental material is available online.
From the Division of Cardiac and Thoracic Surgery $^{\mathrm{a}}$ and the Department of Physiology, ${ }^{\text {b }}$ Temple University School of Medicine, Philadelphia, Pa; and the Division of Cardiac Surgery, ${ }^{c}$ University of Michigan Medical School, Ann Arbor, Mich.

Read at the Eighty-sixth Annual Meeting of The American Association for Thoracic Surgery, Philadelphia, Pa, April 29-May 3, 2006

Received for publication May 22, 2006; revisions received Sept 26, 2007; accepted for publication Oct 2, 2007.

Address for reprints: Mahender Macha, MD, 5144 Cardiovascular Center/5864, University of Michigan Medical Center, 1500 E. Medical Center Dr, Ann Arbor, MI 481095864 (E-mail: mmacha@med.umich.edu).

J Thorac Cardiovasc Surg 2008;135:292-9 $0022-5223 / \$ 34.00$

Copyright $(0) 2008$ by The American Association for Thoracic Surgery

doi:10.1016/j.jtcvs.2007.10.003
Objectives: In a rat model of pressure overload hypertrophy, we studied the effects of intracoronary delivery of mesenchymal stem cells on hemodynamic performance, exercise capacity, systemic inflammation, and left ventricular reverse remodeling.

Methods: Sprague-Dawley rats underwent aortic banding and were followed up by echocardiographic scanning. After a decrease in fractional shortening of $25 \%$ from baseline, animals were randomized to intracoronary injection of mesenchymal stem cells (MSC group; $\mathrm{n}=28$ ) or phosphate-buffered saline solution (control group; $\mathrm{n}$ $=20$ ). Hemodynamic and echocardiographic assessment, swim testing to exhaustion, and measurement of inflammatory markers were performed before the rats were humanely killed on postoperative day 7, 14, 21, or 28 .

Results: Injection of mesenchymal stem cells improved systolic function in the MSC group compared with the control group (mean \pm standard deviation: maximum $\mathrm{dP} / \mathrm{dt}$ $3048 \pm 230 \mathrm{~mm} \mathrm{Hg} / \mathrm{s}$ vs $2169 \pm 97 \mathrm{~mm} \mathrm{Hg} / \mathrm{s}$ at 21 days and $3573 \pm 741 \mathrm{~mm} \mathrm{Hg} / \mathrm{s}$ vs $1363 \pm 322 \mathrm{~mm} \mathrm{Hg} / \mathrm{s}$ at 28 days: $P<.001)$. Time to exhaustion was similarly increased in the MSC group compared with controls (487 \pm 35 seconds vs $306 \pm 27$ seconds at 28 days; $P<.01$ ). Serum levels of interleukins 1 and 6 , tumor necrosis factor-alpha, and brain natriuretic peptide-32 were significantly decreased in animals treated with mesenchymal stem cells. Stem cell transplantation improved left ventricular fractional shortening at 21 and 28 days. Left ventricular end-systolic and enddiastolic diameters were also improved at 28 days.

Conclusions: In this model of pressure overload hypertrophy, intracoronary delivery of mesenchymal stem cells during heart failure was associated with an improvement in hemodynamic performance, maximal exercise tolerance, systemic inflammation, and left ventricular reverse remodeling. This study suggests a potential role of this treatment strategy for the management of hypertrophic heart failure resulting from pressure overload.

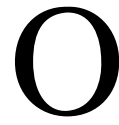
ver the past several years, stem or progenitor cell transplantation therapy has emerged as a promising novel strategy for regenerating lost or damaged myocardium. ${ }^{1,2}$ Several cell types have been proposed as potential donors: skeletal myoblasts, unfractionated or fractionated adult bone marrow stem cells (hematopoietic, mesenchymal, and endothelial progenitor cells), embryonic stem cells, and more recently, resident cardiac progenitor cells. ${ }^{2}$ Controversy exists regarding the possible mechanisms of action responsible for the functional and morphologic improvements observed with various progenitor cell therapies. ${ }^{2,3}$ Although stem cell 

Abbreviations and Acronyms
BNP-32 = brain natriuretic peptide
BrdU = bromodeoxyuridine
ELISA = enzyme-linked immunosorbent assay
GFP $=$ green fluorescent protein
$\mathrm{LV}=$ left ventricular
LVEDD = left ventricular end-diastolic diameter
LVEDP $=$ left ventricular end-diastolic pressure
LVESD = left ventricular end-systolic diameter
LVESP = left ventricular end-systolic pressure
MSC = mesenchymal stem cell
PBS = phosphate-buffered saline solution

differentiation and/or fusion may be responsible, secondary paracrine mechanisms mediated by transplanted cells may lead to more important effects such as neovascularization and angiogenesis, recruitment and expansion of resident cardiac stem cells, or reduced apoptosis.

The adult bone marrow provides an excellent and practical source for autologous stem cells. Both hematopoietic $\left(\mathrm{CD}_{4}{ }^{+} / \mathrm{CD} 33^{+}\right)$and mesenchymal $\left(\mathrm{CD} 34^{-} / \mathrm{CD} 133^{-}\right)$ bone marrow-derived stem cells (MSCs) have demonstrated efficacy in animal and early human studies of heart failure. ${ }^{4}$ In an experimental animal model of myocardial infarction, hematopoietic stem cells have been found to generate de novo myocardium and improve hemodynamic performance. ${ }^{5}$ In 1999, Makino and associates ${ }^{6}$ isolated a cardiomyogenic cell line from mouse bone marrow-derived mesenchymal cells that could be induced to differentiate into cardiomyocytes in vitro by the demethylating agent 5-azacytidine. These cells connected with adjoining cells, formed myotube-like structures, and beat synchronously after 3 weeks in culture. Studies in animal models of ischemic injury have shown that transplantation of 5-azacytidine treated bone marrow-derived MSCs formed cardiac-like muscle cells and improved global contractile function. ${ }^{7,8}$ Most attempts to study the potential of stem cell transplantation in heart failure have been centered on ischemic heart disease. Nonischemic cardiomyopathy, including cardiac hypertrophy resulting from pressure overload, is also a common cause of end-stage heart failure. Stem cell transplantation may offer a novel therapeutic option as an adjunct to surgical therapy in valvular and nonischemic cardiomyopathy. In a rat model of pressure overload hypertrophy in transition to heart failure, we studied the hemodynamic and molecular effects of intracoronary delivery of bone marrow-derived MSCs. We hypothesized that bone marrow-derived MSC transplantation would improve ventricular function and exercise capacity and that these functional benefits would be associated with an amelioration in profiles of systemic inflammation, volume overload, and left ventricular (LV) remodeling.

\section{Materials and Methods}

All animals received humane care in compliance with institutional guidelines and the "Guide for the Care and Use of Laboratory Animals" prepared by the Institute of Laboratory Animal Resources, Commission on Life Sciences, National Research Council.

\section{Experimental Model of Heart Failure}

Outbred male Sprague-Dawley rats (200-250 g, Harlan, Indianapolis, Ind) were anesthetized, endotracheally intubated, and mechanically ventilated. An upper median sternotomy was performed and a small titanium clip with an internal diameter of $0.6 \mathrm{~mm}$ (Ligaclip Extra; Ethicon Endo-Surgery, Inc, Cincinnati, Ohio) was applied across the aorta proximal to the right brachiocephalic artery. All animals underwent baseline echocardiography 10 weeks after clip placement. Transthoracic M-mode and 2-dimensional echocardiography was performed with a Hewlett-Packard Sonos 5500 Imaging System (Hewlett-Packard, Palo Alto, Calif) using a 4-MHz transducer. Images were standardized to short-axis view at the LV midpapillary level, and end-systolic diameter (LVESD), end-diastolic diameter (LVEDD), and fractional shortening were recorded. Starting at 10 weeks after aortic clip placement, echocardiography was performed weekly. After detection of an absolute decline in fractional shortening of $25 \%$ from baseline (relative $50 \%$ reduction), animals were randomized to intracoronary delivery of bone marrow-derived MSCs (MSC group, $\mathrm{n}=28$ ), or phosphate-buffered saline solution (PBS; control group, $\mathrm{n}=20$ ).

\section{Isolation of Bone Marrow-derived MSC and Culture Technique}

Primary cultures of MSCs were obtained from the femurs and tibias of adult male Sprague-Dawley rats (Taconic Farms, Germantown, NY) as described previously. ${ }^{9}$ In brief, rats were humanely killed with a mixture of $70 \%$ carbon dioxide and $30 \%$ oxygen. Tibias and femurs were removed and placed on ice in complete medium containing minimal essential medium with alpha modification ( $\alpha$ MEM; Gibco BRL, Life Technologies, Gaithersburg, Md) with $20 \%$ fetal calf serum (Atlanta Biologicals, Norcross, Ga), L-glutamine $(2 \mathrm{mmol} / \mathrm{L})$, penicillin (100 units $/ \mathrm{mL})$, streptomycin $(100$ $\mu \mathrm{g} / \mathrm{mL})$, and amphotericin B (25 ng/mL; Mediatech, Herndon, Va). Under sterile conditions, a 21-gauge needle attached to a 10$\mathrm{mL}$ syringe filled with medium was used to flush out the marrow. Bone marrow was filtered through a 70- $\mu$ m nylon mesh and plated in a $75-\mathrm{cm}^{2}$ flask (Becton Dickinson, Franklin Lakes, NJ). MSCs were isolated by their adherence to plastic. About 24 hours after being plated, nonadherent cells were removed and fresh medium was added. After the cells had grown to near confluency, they were passaged 2 to 6 times by being detached $(0.25 \%$ trypsin $/ 1 \mathrm{mmol} / \mathrm{L}$ ethylenediaminetetraacetic acid for 5 minutes) and replated at a density of around 5000 cells $/ \mathrm{cm}^{2}$ (high-density conditions). At this point cells were frozen in vials containing approximately 500,000 cells per unit. Preliminary studies with reverse-transcriptase polymerase chain reaction revealed that these cells expressed GATA-4 and Nkx2.5, which are early cardiac lineage markers (data not shown). Five days before intracoronary cell delivery in rats, MSCs were thawed and cultured in 10\% $\alpha$-MEM for 24 hours. The second day, MSCs were plated and $10 \%$ fresh Iscove's modified Dulbecco's medium was added. In addition, bromodeoxyuridine (BrdU) was supplemented to the culture medium at a concentration of 5 
$\mu \mathrm{mol} / \mathrm{L}$ for 24 hours. On the third day, 5-azacytidine was added to the culture medium at a concentration of $10 \mu \mathrm{g} / \mathrm{mL}$ for 24 hours. Culture medium was then replaced with fresh $10 \%$ Iscove's modified Dulbecco's medium on the fourth day. Finally, on the fifth day, MSCs were trypsinized and were ready for injection. One hundred fifty microliters of a solution containing $1 \times 10^{6}$ MSCs in PBS or PBS alone was mixed with $50 \mu \mathrm{L}$ of adenosine just before injection. We chose this particular cell dose on the basis of prior studies of MSC and myoblast transplantation, which have demonstrated the safety and efficacy of intracoronary infusion of 1 million cells. ${ }^{10,11}$

\section{MSC Delivery}

Within 48 hours of detection of more than $25 \%$ decrease in fractional shortening from baseline and subsequent randomization, animals were anesthetized and a full median sternotomy was performed. The aorta and main pulmonary artery were encircled together with 4-0 Prolene polypropylene (Ethicon, Inc, Somerville, $\mathrm{NJ}$ ) and a tourniquet was loosely applied. With the use of a 1-mL syringe attached to a sterile 30 -gauge needle, the solution containing $1 \times 10^{6}$ MSCs in PBS and adenosine, or PBS and adenosine alone, was injected into the aortic root during brief occlusion of the aorta and the pulmonary artery (5 seconds). Intracoronary delivery was confirmed by temporary observation of bradycardia and epicardial blanching.

\section{MSC Identification and Cell Counting After Intracoronary Infusion}

Additional animals were used to confirm successful delivery of MSCs. Animals were humanely killed by cardiectomy 2 hours $(n=4), 3$ days $(n=4), 7$ days $(n=4)$, and 21 days $(n=4)$ after intracoronary delivery of MSCs. The hearts were thoroughly washed with PBS cooled to $4^{\circ} \mathrm{C}$. After resection of the atria, the ventricles were fixed in $10 \%$ formalin for 24 hours. Hearts were embedded in paraffin and cut into slices of $4-\mu \mathrm{m}$ thickness. Monoclonal antibodies against BrdU (Zymed BrdU staining kit, San Francisco, Calif) were used to identify transplanted cells. For each animal at each time point, 10 separate heart sections were examined under $400 \times$ magnification with an E600 Nikon microscope (Nikon, Tokyo, Japan). The number of BrdU-labeled cells per section was quantified by the BIOQUANT Life Science Core Analysis System (Bioquant Image Analysis Corporation, Nashville, Tenn).

\section{Echocardiographic Assessment and Hemodynamic Measurement}

Within the treatment groups, animals were randomly assigned to be humanely killed on postoperative day $7,14,21$, or 28 (5 animals at each time interval in the control group and 7 animals at each time interval in the MSC group). Animals were anesthetized and echocardiographic assessment was performed. Images were standardized to short-axis view at the LV midpapillary level, and fractional shortening, LVESD, LVEDD, and LV free wall diameter were recorded. Subsequently, a full median sternotomy was performed. A 21-gauge intravenous catheter connected to a Digi-Med System Integrator model 200 (Micro-Med, Louisville, Ky) was advanced from the ventricular apex into the LV cavity. Heart rate, LV end-systolic pressure (LVESP), LV end-diastolic pressure (LVEDP), time constant of isovolumetric pressure decay (tau), rate of rise of LV pressure $\left(\mathrm{dP} / \mathrm{dt}_{\max }\right)$, and rate of fall of $\mathrm{LV}$ pressure $\left(\mathrm{dP} / \mathrm{dt}_{\min }\right)$ were recorded.
Five milliliters of blood was then withdrawn for enzyme-linked immunosorbent assay (ELISA) analysis. Animals were humanely killed by cardiectomy.

\section{Exercise Testing}

All animals underwent swim testing to exhaustion 1 day before being killed. As described by Musch and colleagues, ${ }^{12}$ this protocol has been shown to cause a rat to use $65 \%$ to $70 \%$ of its maximum oxygen consumption. Exhaustion was defined as the time at which the animal was no longer able to consistently keep its head above the water level. Investigators remained blinded to the treatment groups throughout the experiments to avoid bias.

\section{ELISA Analysis}

Blood samples were allowed to clot for 2 hours at room temperature before centrifugation for 20 minutes at approximately $2000 \mathrm{~g}$. The serum was removed, an aliquot was obtained, and it was stored at $-70^{\circ} \mathrm{C}$. Cytokines and cachectins were quantified with the following ELISA kits: Quantikine Rat IL-1 beta (R\&D Systems, Minneapolis, Minn), Quantikine Rat IL-6, Quantikine Rat TNF-alpha, and Rat BNP-32 Immunoassay Kit (Peninsula Laboratories, San Carlos, Calif). Optical density was obtained within 30 minutes by an MRX Dynatech Laboratories microplate reader (Dynatech Laboratories, Inc, Foster City, Calif) and Revelation version 1.01 software (Revelation Software, London, United Kingdom). Readings were taken at $540 \mathrm{~nm}$ and subtracted from readings at $450 \mathrm{~nm}$ to correct for optical imperfections in the plates. Duplicate readings for each sample, control, and standard were averaged, subtracting out the average zero standard optical density. A standard curve was constructed by plotting the mean absorbance for each standard in the y-axis against the concentration on the $\mathrm{x}$-axis with a best fit curve drawn through the points on the graph.

\section{Statistical Analysis}

All data are presented as mean \pm standard deviation. Betweengroup differences in frequency data were analyzed by the Fisher exact test. Differences in continuous variables were analyzed by independent $t$ tests. Analysis of variance with least significant difference procedure was used. After analysis of variance, a Bonferroni post hoc analysis was used for adjustment for multiple comparisons. The SAS v8.1 software (SAS Institute, Inc, Cary, NC) was used for statistical analysis.

\section{Results}

\section{Development of Heart Failure}

A decline of $25 \%$ of fractional shortening after aortic banding compared with baseline was detected between 175 and 237 days after clip placement for all animals (mean \pm SD: $203 \pm 15$ days). There were no statistical differences between treatment groups $(202 \pm 14$ days vs $205 \pm 18$ days for the MSC and control groups, respectively; $P=.55$ ). Fractional shortening was also similar between animals in the MSC and control groups at baseline echocardiographic assessment $(51.2 \% \pm 1.7 \%$ vs $51.0 \% \pm 2.2 \%: P=.71)$ and treatment randomization $(26.5 \% \pm 1.7 \%$ vs $26.0 \% \pm 2.1 \% ; P=.41)$. We observed a combined $30 \%$ mortality rate during aortic clip placement and development of heart failure. 
TABLE 1. Hemodynamic parameters at 7, 14, 21, and 28 days after treatment

\begin{tabular}{|c|c|c|c|c|c|c|c|c|}
\hline & \multicolumn{2}{|c|}{7 Days } & \multicolumn{2}{|c|}{14 Days } & \multicolumn{2}{|c|}{21 Days } & \multicolumn{2}{|c|}{28 Days } \\
\hline & Control & MSC & Control & MSC & Control & MSC & Control & MSC \\
\hline HR (beats/min) & $326 \pm 32$ & $316 \pm 41$ & $324 \pm 40$ & $311 \pm 32$ & $323 \pm 40$ & $315 \pm 39$ & $298 \pm 33$ & $286 \pm 29$ \\
\hline LVESP $(\mathrm{mm} \mathrm{Hg})$ & $112 \pm 16$ & $114 \pm 15$ & $105 \pm 12$ & $111 \pm 13$ & $99 \pm 10$ & $115 \pm 11^{*}$ & $91 \pm 9$ & $122 \pm 14^{*}$ \\
\hline LVEDP $(\mathrm{mm} \mathrm{Hg})$ & $16 \pm 4$ & $15 \pm 3$ & $17 \pm 5$ & $14 \pm 4$ & $19 \pm 5$ & $12 \pm 4$ & $22 \pm 4$ & $11 \pm 3^{*}$ \\
\hline Tau (ms) & $26 \pm 5$ & $25 \pm 4$ & $28 \pm 4$ & $25 \pm 3$ & $29 \pm 5$ & $23 \pm 3$ & $31 \pm 3$ & $22 \pm 2^{*}$ \\
\hline
\end{tabular}

MSC, Mesenchymal stem cell; HR, heart rate; $L V E S P$, left ventricular end-systolic pressure; LVEDP; left ventricular end-diastolic pressure; tau, the time constant of isovolumetric pressure decay. ${ }^{*} P<.05$ for comparison of control group versus MSC group.

Additionally, 4 animals died after treatment ( 3 in the control group and 1 in the MSC group).

\section{MSC Identification and Cell Counting After Intracoronary Infusion}

Figure E1 shows a representative section of LV myocardium 2 hours after intracoronary delivery of MSCs. Numerous BrdU-labeled MSCs (brown nuclei) are seen by light microscopy $(400 \times)$. Some of these cells are visualized within or around capillary vessels. Blue-stained nuclei correspond to cardiomyocytes and endothelial cells. Figure E2 shows a section of myocardium 21 days after MSC transplantation. Although the intensity of the brown staining has decreased, several BrdU-positive nuclei can be distinguished among blue-stained nuclei of native cardiomyocytes. We found the following average number $( \pm \mathrm{SD})$ of BrdU-positive cells per high-power field at each time interval: $205 \pm 74$ cells at 2 hours, $195 \pm 44$ cells at 3 days, $180 \pm 64$ cells at 7 days, and $38 \pm 23$ cells at 21 days.

\section{Hemodynamic Assessment}

Hemodynamic parameters were measured at similar heart rates for both groups (Table 1). The control group showed a decline in LVESP and a rise in LVEDP with progression of heart failure. The MSC group, however, demonstrated significantly increased LVESP and reduced LVEDP compared with the control group at 28 days after treatment. The time constant of isovolumetric pressure decay (tau) was found to be prolonged in controls and decreased in animals receiving MSCs at 28 days. As shown in Figure 1, animals receiving MSCs revealed stabilization of LV systolic function (dP/ $\left.\mathrm{dt}_{\max }\right)$ after treatment. However, control animals revealed a steady decline of systolic function as heart failure progressed. The differences were significant at 21 days and 28 days. Similar findings were demonstrated with diastolic relaxation $\left(\mathrm{dP} / \mathrm{dt}_{\min }\right)$, as shown in Figure E3.

\section{Exercise Test}

Both groups of animals were able to swim for longer periods of time as they recovered from the median sternotomy procedure at day 0. However, animals receiving MSCs demonstrated improved exercise capacity at all time intervals in comparison with the control group (Figure 2). These differences were statistically significant at 21 and 28 days.

\section{Systemic Inflammation and Volume Overload}

Serum levels of systemic inflammatory markers are shown in Table 2. Levels of interleukins 1 and 6 and tumor necrosis factor- $\alpha$ were decreased in MSC-treated animals at every time interval in comparison with the control group, which demonstrated increasing levels as heart failure progressed. These differences were significant at 21 and 28 days. Levels of brain natriuretic peptide-32 (BNP-32), also shown in Table 2, were remarkably decreased in the cell treatment group at 21 and 28 days compared with the control group.

\section{Echocardiographic Assessment}

Control animals revealed a steady decline of fractional shortening as heart failure progressed. The MSC group, however, demonstrated stabilization of fractional shortening and a slight improvement at 28 days. These differences were statistically significant at 21 and 28 days (Figure 3). Control animals showed a gradual increase of LVESD as heart failure progressed. The MSC group revealed stabilization of LV dilatation and a slight reduction of this diameter at 28 days. These differences were again statistically significant at 21 and 28 days (Figure 4). LVEDD was found to increase in control animals with progression of heart failure. The cell transplantation group, however, demonstrated early stabilization and subsequent improvement (Figure 5). Control animals showed a decline of free wall diameter as heart failure progressed. Animals receiving cells demonstrated less reduction of this diameter. These differences were statistically significant at 21 days only (Figure E4).

\section{Discussion}

The use of stem cell populations to improve the structure and function of the damaged heart is an exciting and controversial area, generating great debate over the efficacy and mechanism of action of injected cells. ${ }^{1,2}$ Most attempts have been centered in animal models of ischemic heart disease. To date, only two studies have examined the potential beneficial effects of bone marrow-derived stem cell transplantation in nonischemic animal models. Agbulut and coworkers ${ }^{13}$ 


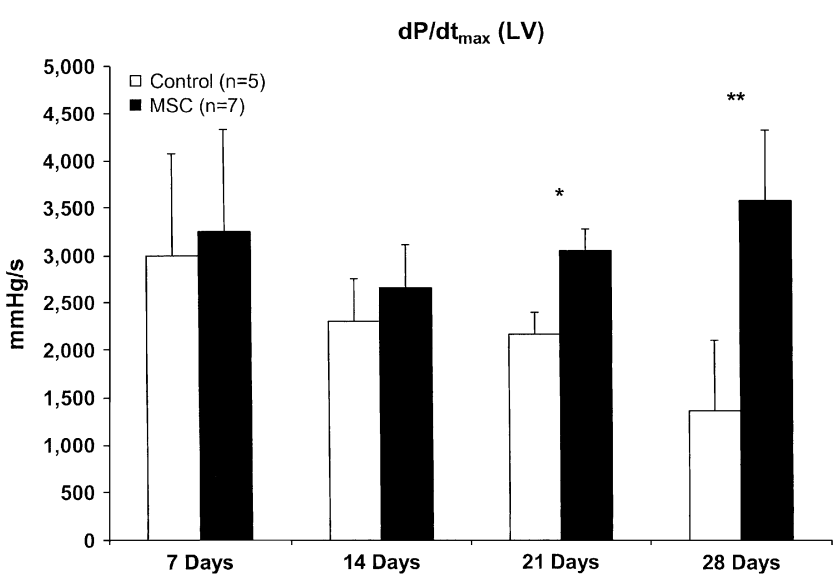

Figure 1. Left ventricular maximal $\mathrm{dP} / \mathrm{dt}$ at each time interval. ${ }^{*} \boldsymbol{P}<.05$ and ${ }^{* *} \boldsymbol{P}<.001 \mathrm{MSC}$ versus control group. MSC, Mesenchymal stem cell.

showed myogenic differentiation of transplanted unpurified bone marrow cells in a murine model of doxorubicin-induced dilated cardiomyopathy. However, the authors questioned the potential functional efficacy of this finding because of the very low number of transdifferentiated cells they observed. Recently, Nagaya and associates ${ }^{14}$ reported improved cardiac function after MSC transplantation in a rat model of myocarditis leading to dilated cardiomyopathy. To our knowledge, no studies have evaluated the efficacy of stem cell transplantation therapy in an animal model of pressure overload-induced heart failure.

The degree of functional improvement observed in the present study after bone marrow-derived MSC transplantation is remarkable. Control animals showed a continuous decline in systolic and diastolic function as heart failure progressed after treatment with PBS. Animals in the MSC group, however, demonstrated stabilization of LV contractility and improved ventricular relaxation after cell transplantation therapy. These findings were most remarkable at 21 and 28 days after treatment. Similarly, we report a significant improvement in exercise capacity in the MSC group compared with controls. As the median sternotomy wound progressively healed, both animal groups showed better exercise tolerance. This improvement was also observed in the control group, despite a progressive decline in systolic and diastolic function. However, animals that received MSCs demonstrated significantly improved exercise performance compared with controls at later intervals. Although an improvement in exercise capacity after cell transplantation therapy in heart failure is not a novel finding, ${ }^{15}$ we believe this is a remarkable observation in this animal model in transition to advanced heart failure.

It has been demonstrated that progression of heart failure is associated with upregulation of tumor necrosis factor- $\alpha$, inter-

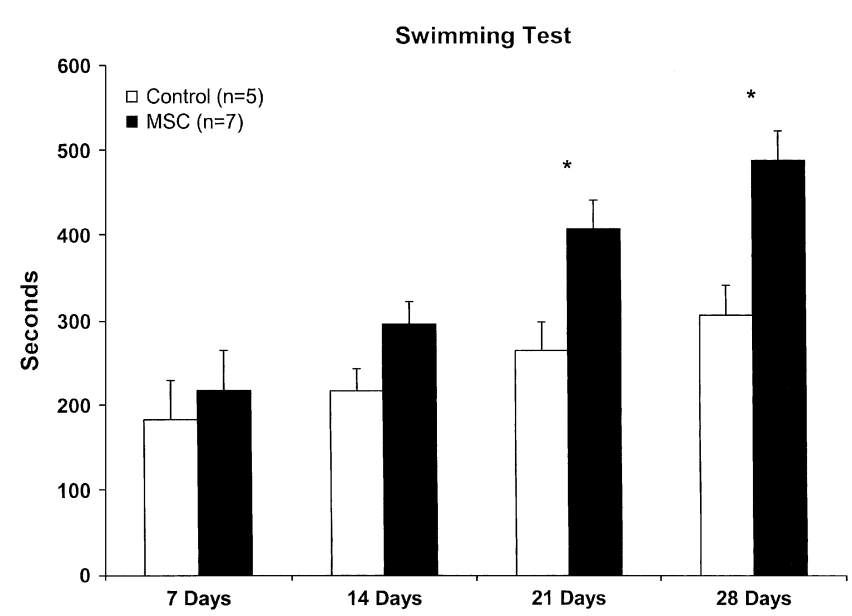

Figure 2. Maximal exercise tolerance at each time interval. ${ }^{*} \boldsymbol{P}<.01$ MSC versus control group. MSC, Mesenchymal stem cell.

leukin 1, and interleukin 6 plasma levels. These mediators activate several key pathways in the cell that enhance matrix remodeling, promote apoptosis and depress cardiac contractility. ${ }^{16}$ In this study, we report a remarkable decline in tumor necrosis factor- $\alpha$, interleukin 1 , and interleukin 6 serum levels in the cell treatment group at 21 and 28 days. These findings support the hypothesis that MSC treatment ameliorates the inflammatory response that is associated with progression of heart failure. However, this study was not designed to provide any specific mechanistic insight to reveal the biological effects of MSCs on these different pathways.

BNP-32 is a cardiac neurohormone specifically secreted by ventricular myocytes in response to ventricular volume expansion, pressure overload, and increased wall tension. A decline in BNP-32 serum levels is a useful indicator of response

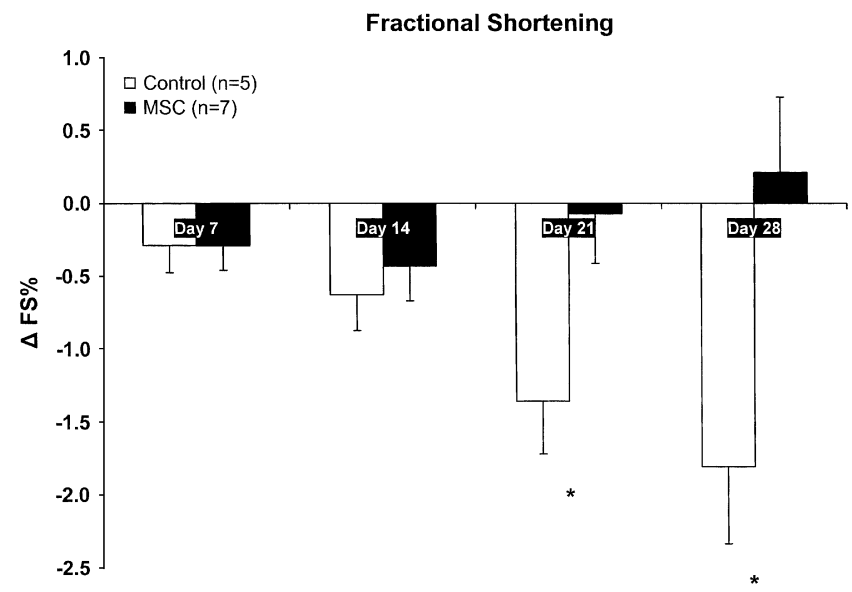

Figure 3. Changes in fractional shortening (FS) from day $\mathbf{0}$ at each time interval. ${ }^{*} \boldsymbol{P}<.01$ MSC versus control group. MSC, Mesenchymal stem cell. 
TABLE 2. Serum levels $(\mathrm{pg} / \mathrm{mL})$ of inflammatory markers and BNP-32 at 7, 14, 21, and 28 days after treatment

\begin{tabular}{|c|c|c|c|c|c|c|}
\hline & \multicolumn{3}{|c|}{7 Days } & \multicolumn{3}{|c|}{14 Days } \\
\hline & Control & MSC & $\% \Delta$ & Control & MSC & $\% \Delta$ \\
\hline IL-1 & $34.22 \pm 1.76$ & $33.37 \pm 1.49$ & -2.5 & $36.12 \pm 1.51$ & $34.46 \pm 0.58$ & -4.6 \\
\hline IL-6 & $179.84 \pm 15.7$ & $173.21 \pm 6.2$ & -3.7 & $190.94 \pm 8.9$ & $179.73 \pm 4.7$ & -5.9 \\
\hline TNF- $\alpha$ & $15.88 \pm 2.55$ & $15.04 \pm 1.83$ & -5.3 & $17.76 \pm 2.20$ & $16.24 \pm 1.33$ & -8.6 \\
\hline BNP-32 & $23.92 \pm 6.10$ & $23.11 \pm 5.30$ & -3.4 & $28.08 \pm 3.95$ & $25.73 \pm 3.98$ & -8.4 \\
\hline
\end{tabular}

$B N P$, Brain natriuretic peptide; $M S C$, mesenchymal stem cell; $I L$, interleukin; $T N F$, tumor necrosis factor. ${ }^{*} P<.05$ for comparison of control group versus MSC group.

to medical therapy in patients with end-stage heart failure. ${ }^{17}$ The remarkable decline in serum levels of BNP-32 that we observed in the MSC treatment group demonstrates an amelioration of the volume overload state associated with advanced heart failure.

In the present study, we also report echocardiographic signs of reverse remodeling in animals receiving MSCs, such as improved fractional shortening, decreased LVESD and LVEDD, and improvement of LV free wall diameter. Although several studies have shown beneficial effects of cell transplantation therapy on fractional shortening and LV diameters in animal models of heart failure, ${ }^{10,14,18,19}$ echocardiographic signs of reverse remodeling have not been previously reported in this model of pressure overload hypertrophy. The moderate improvement we observed on these echocardiographic parameters was seen after a single dose of 1 million MSCs. It is unknown whether higher doses or repeated doses of MSCs would lead to a significantly better improvement. Dose-response testing studies with longer follow-up intervals are needed.

The improvement in hemodynamic performance and exercise capacity we observed, and the echocardiographic signs of reverse remodeling associated with these beneficial functional effects, correlated histologically with the pres-

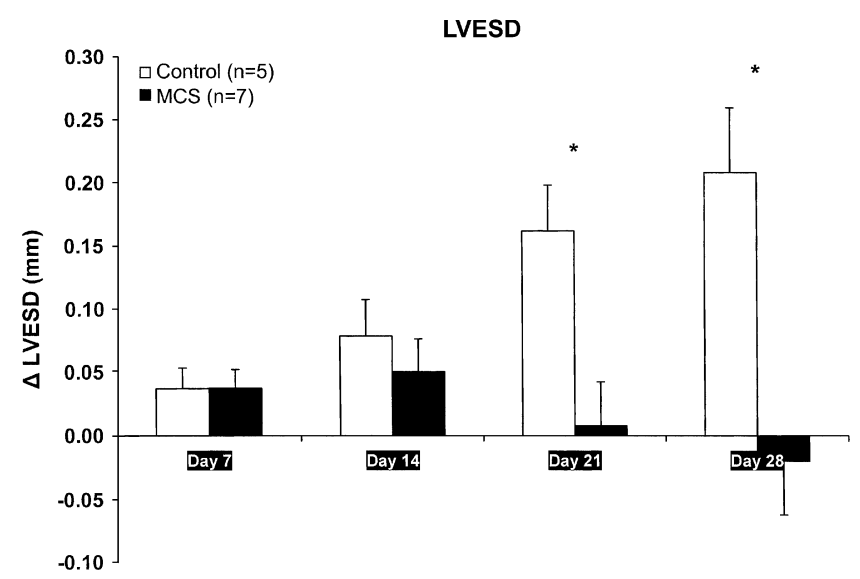

Figure 4. Changes in left ventricular end-systolic diameter (LVESD) from day 0 at each time interval. ${ }^{*} P<.01$ MSC versus control group. MSC, Mesenchymal stem cell. ence of BrdU-stained cells at early and late time intervals. A significant percentage of the number of BrdU-stained cells visualized immediately after delivery ( 2 hours) were present at 3 days (95\%) and 7 days (88\%). The percentage of BrdU-positive cells observed at 21 days was still significant $(18 \%)$. Whether these cells represent MSCs, transdifferentiated cardiomyocytes, or fused cells is unknown. However, these findings demonstrate that a significant number of cells visualized immediately after intracoronary delivery are able to survive and may play a significant biological role 21 days later, when most of the functional and molecular beneficial findings we reported are manifested. It is reasonable to speculate that some of these biological effects may be mediated by paracrine mechanisms. A hypothesis that is growing in interest is that MSCs may be able to ameliorate the local inflammatory response, promote angiogenesis, improve the myocardial apoptotic profile, and promote reverse remodeling through the action of growth factors. $^{4,20}$ Currently, we are investigating the LV tissue levels of key growth factors such as vascular endothelial growth factor, insulin-like growth factor, hepatocyte growth factor, and platelet-derivated growth factor after cell transplantation in this model.

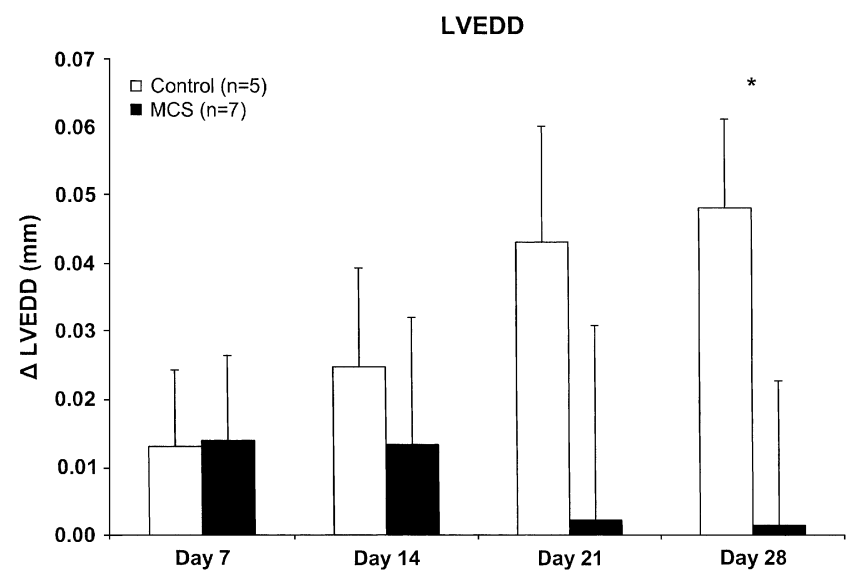

Figure 5. Changes in left ventricular end-diastolic diameter (LVEDD) from day 0 at each time interval. ${ }^{*} P<.01$ MSC versus control group. MSC, Mesenchymal stem cell. 
TABLE 2. Continued

\begin{tabular}{|c|c|c|c|c|c|}
\hline \multicolumn{3}{|c|}{21 Days } & \multicolumn{3}{|c|}{28 Days } \\
\hline Control & MSC & $\% \Delta$ & Control & MSC & $\% \Delta$ \\
\hline $37.15 \pm 0.76$ & $33.86 \pm 0.63^{*}$ & -8.6 & $41.08 \pm 1.36$ & $32.40 \pm 1.17^{*}$ & -21.1 \\
\hline $205.80 \pm 5.7$ & $171.34 \pm 2.0^{*}$ & -16.8 & $228.58 \pm 8.2$ & $167.83 \pm 6.0^{*}$ & -26.6 \\
\hline $19.38 \pm 0.61$ & $15.59 \pm 0.67^{*}$ & -19.6 & $26.10 \pm 1.99$ & $14.64 \pm 1.20^{*}$ & -43.9 \\
\hline $30.08 \pm 0.26$ & $21.11 \pm 1.80^{*}$ & -29.8 & $36.85 \pm 3.24$ & $19.84 \pm 3.08 *$ & -46.2 \\
\hline
\end{tabular}

To our knowledge, this study demonstrates for the first time beneficial effects of MSC transplantation therapy in a model of pressure overload hypertrophy. On the basis of these results, we speculate that patients with aortic stenosis or hypertension in transition to heart failure may potentially benefit from bone-derived MSC transplantation therapy (combined with aortic valve replacement, for example). However, we should be very cautious about the clinical implications of this study. Additional small animal studies should confirm these results. Studies in large animal models should also be conducted before entering into the clinical arena. Many important questions need to be answered first: Are MSCs the optimal cell population? What is the best method of delivery? What is the most effective dose? Is a single dose effective enough or are multiple doses required to achieve a sustained response? Do cells need to be treated with 5-azacytidine or other inductor of differentiation? Are these inductors safe? Do MSCs have significant side effects? Do bone marrow-derived MSCs from older patients have the same therapeutic potential as cells from younger patients? What is the role of combined therapies?

In conclusion, intracoronary delivery of MSCs during heart failure in this model of pressure overload hypertrophy improved hemodynamic performance and maximal exercise tolerance. MSC transplantation was also associated with improvement in markers of systemic inflammation, volume overload, and echocardiographic signs of LV reverse remodeling. These effects were most remarkable at 21 and 28 days after injection. This study suggests a potential role of this treatment strategy for the management of hypertrophic heart failure resulting from pressure overload.

We express sincere gratitude to Dr S. Azizi, Dr B. Krynska, and B. Augelli (Neurology Department, Temple University School of Medicine) for providing mesenchymal stem cells and continuous technical support throughout this study.

\section{References}

1. Murry CE, Field LJ, Menasché P. Cell based cardiac repair: reflections at the 10-year point. Circulation. 2005;112:3174-83.

2. Wollert KC, Drexler H. Clinical applications of stem cells for the heart. Circ Res. 2005;96:151-63.

3. Dimmeler S, Zeiher AM, Schneider MD. Unchain my heart: the scientific foundations of cardiac repair. J Clin Invest. 2005;115:572-83.
4. Leri A, Kajstura J, Anversa P. Cardiac stem cells and mechanisms of myocardial regeneration. Physiol Rev. 2005;85:1373-416.

5. Orlic D, Kajstura J, Chimenti S, Jakoniuk I, Anderson SM, Li B, et al. Bone marrow cells regenerate infarcted myocardium. Nature. 2001; 410:701-5.

6. Makino S, Fukuda K, Miyoshi S, Konishi F, Kodama H, Pan J, et al. Cardiomyocytes can be generated from marrow stromal cells in vitro. J Clin Invest. 1999;103:697-705.

7. Tomita S, Li RK, Weisel RD, Mickle DA, Kim EJ, Sakai T, et al. Autologous transplantation of bone marrow cells improves damaged heart function. Circulation. 1999;100(19 Suppl):II247-56.

8. Tomita S, Mickle DA, Weisel RD, Jia ZQ, Tumiati LC, Allidina Y, et al. Improved heart function with myogenesis and angiogenesis after autologous porcine bone marrow stromal cell transplantation. $J$ Thorac Cardiovasc Surg. 2002;123:1132-40.

9. Schwarz EJ, Alexander GM, Prockop DJ, Azizi SA. Multipotential marrow stromal cells transduced to produce L-DOPA: engraftment in a rat model of Parkinson's disease. Hum Gene Ther. 1999;10:2539-49.

10. Saito T, Kuang JQ, Lin CC, Chiu RC. Transcoronary implantation of bone marrow stromal cells ameliorates cardiac function after myocardial infarction. J Thorac Cardiovasc Surg. 2003;126:114-23.

11. Suzuki K, Murtuza B, Suzuki N, Smolenski RT, Yacoub MH. Intracoronary infusion of skeletal myoblasts improves cardiac function in doxorubicin-induced heart failure. Circulation. 2001;104:213-7.

12. Musch TI, Ghaul MR, Tranchitelle S, Zelis R. Skeletal muscle depletion during submaximal exercise in rats with chronic heart failure. Basic Res Cardiol. 1990;85:606-18.

13. Agbulut O, Menot ML, Li Z, Marotte F, Paulin D, Hagege AA, et al. Temporal patterns of bone marrow cell differentiation following transplantation in doxorubicin-induced cardiomyopathy. Cardiovasc Res. 2003;58:451-9.

14. Nagaya N, Kangawa K, Itoh T, Iwase T, Murakami S, Miyahara Y, et al. Transplantation of mesenchymal stem cells improves cardiac function in a rat model of dilated cardiomyopathy. Circulation. 2005;112:1128-35.

15. Jain M, DerSimonian H, Brenner DA, Ngoy S, Teller P, Edge AS, et al. Cell therapy attenuates deleterious ventricular remodeling and improves cardiac performance after myocardial infarction. Circulation. 2001;103: 1920-7.

16. Kerkela R, Force T. p38 mitogen-activated protein kinase: a future target for heart failure therapy? J Am Coll Cardiol. 2006;48:556-8.

17. Maeda K, Tsutamoto T, Wada A, Hisanaga T, Kinoshita M. Plasma brain natriuretic peptide as a biochemical marker of high left ventricular end-diastolic pressure in patients with symptomatic left ventricular dysfunction. Am Heart J. 1998;135:825-32.

18. Rajnoch C, Chachques JC, Berrebi A, Bruneval P, Benoit MO, Carpentier A. Cellular therapy reverses myocardial dysfunction. J Thorac Cardiovasc Surg. 2001;121:871-8.

19. Silva GV, Litovsky S, Assad JA, Sousa AL, Martin BJ, Vela D, et al. Mesenchymal stem cells differentiate into an endothelial phenotype, enhance vascular density, and improve heart function in a canine chronic ischemia model. Circulation. 2005;111:150-6.

20. Xu M, Uemura R, Dai Y, Wang Y, Pasha Z, Ashraf M. In vitro and in vivo effects of bone marrow stem cells on cardiac structure and function. J Mol Cell Cardiol. 2007;42:441-8. 


\section{Discussion}

Dr Y. Joseph Woo (Philadelphia, $\mathrm{Pa}$ ). You should be congratulated for a fantastic study, particularly with the technical aspects that you showed and also the creative water bath assay. You answered a lot of the potential questions that the audience would have with in your last two slides.

Your study is very good at showing the effects of your therapy. To take your study to the next level, you should show some mechanistic components. The investigators who are doing a lot of the stem cell transplantation work are thinking that there is not much cross-linking of the cells and probably not a tremendous contractile contribution from stem cell transplantation, but rather some sort of biofactory effect. You did measure a multitude of different parameters. Can you comment on some of the other things that you may be in the process of measuring that would perhaps better elucidate the mechanism in effect here?

Dr Molina. Thank you, Dr Woo, for your comments and questions. Regarding the mechanisms of action of bone marrow-derived MSCs, I think that is one of the fundamental questions in this area of research, as you mentioned. The contribution of MSCs to the improvement in contractile function of these hearts is probably mild. Contractile function is improved in treated animals in comparison with the control group at 21 and 28 days after therapy, but if we compare treated animals with baseline contractility before cell transplantation, it is probably similar. We think that both systolic and diastolic function became stable after treatment. Treatment with bone marrow-derived MSCs, at least in this study, avoided the deterioration in contractility that occurs with progression of heart failure. Whether the cells really differentiate into cardiomyocytes is still unknown. We are still running studies to identify these cells at later intervals, and we are planning to do it with confocal microscopy. However, there are other findings that I did not present that are also significant in terms of alternative potential mechanisms of action. We have measured tissue growth factors in these hearts, and several of them are significantly increased in the cell treatment group: for example, insulin-like growth factor, hepatocyte growth factor, and platelet-derived growth factor. We think that the paracrine effects that these cells exert on native cardiomyocytes might play a very significant role in terms of the mechanisms of action.

Dr Beat H. Walpoth (Geneva, Switzerland). Although you stated improvements in remodeling, function, and anti-inflammation, so far as I have seen, you have only shown one slide with a few labeled cells at a very early time point after injection. Did you find any surviving cells at 28 days and did you try to quantify them? Additionally, differentiation and characterization of the injected cells would be helpful to support your hemodynamic results.

Dr Molina. Thank you for your question. These cells were marked not only with BrdU, as I showed in two slides, but also with green fluorescent protein (GFP). Both of these markers tend to disappear with cellular division. By Western blot analysis we were able to find cells positive for GFP only up to 14 days after cell injection. We did not find any GFP after that time interval. As I mentioned before, we are still in the process of obtaining images with confocal microscopy at later time intervals, which represents a challenging task.

In our next studies we are planning to use GFP with a lentiviral GFP adenovirus transfection, which is a much more stable transfection and is going to allow us to trace these cells for longer periods of time.

Dr Mark J. Krasna (Baltimore, Md). Dr Molina, I have one quick question. Technically, you were able to do two sternotomies and a swim time. Did any of these rats die? In the manuscript it appears that they all survived.

Dr Molina. Not all of them survived. In the first operation, aortic clip placement, there was a survival of about $70 \%$. About $15 \%$ of the animals died during or right after the operation, usually of respiratory complications, and another $15 \%$ usually died during the follow-up period as heart failure developed. 


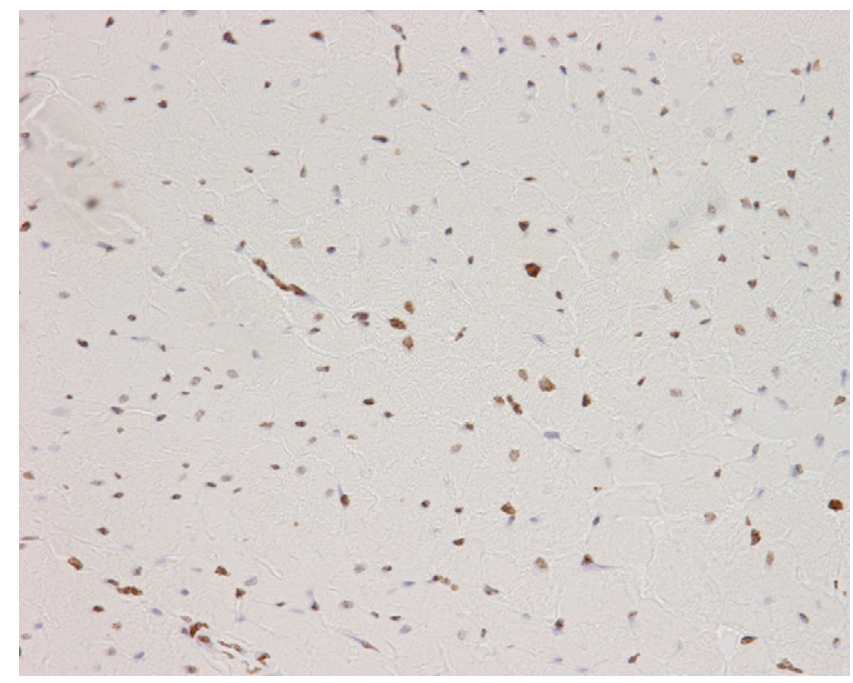

Figure E1. Section of left ventricular myocardium 2 hours after intracoronary delivery of mesenchymal stem cells $(400 \times)$.

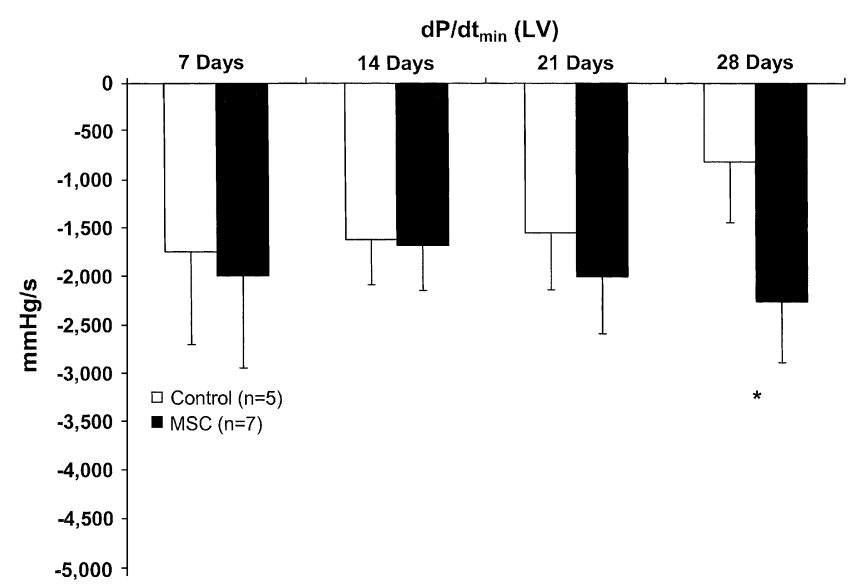

Figure E3. Left ventricular maximal negative $\mathrm{dP} / \mathrm{dt}$ at each time interval. ${ }^{*} \boldsymbol{P}<.01 \mathrm{MSC}$ versus control group. MSC, Mesenchymal stem cell.

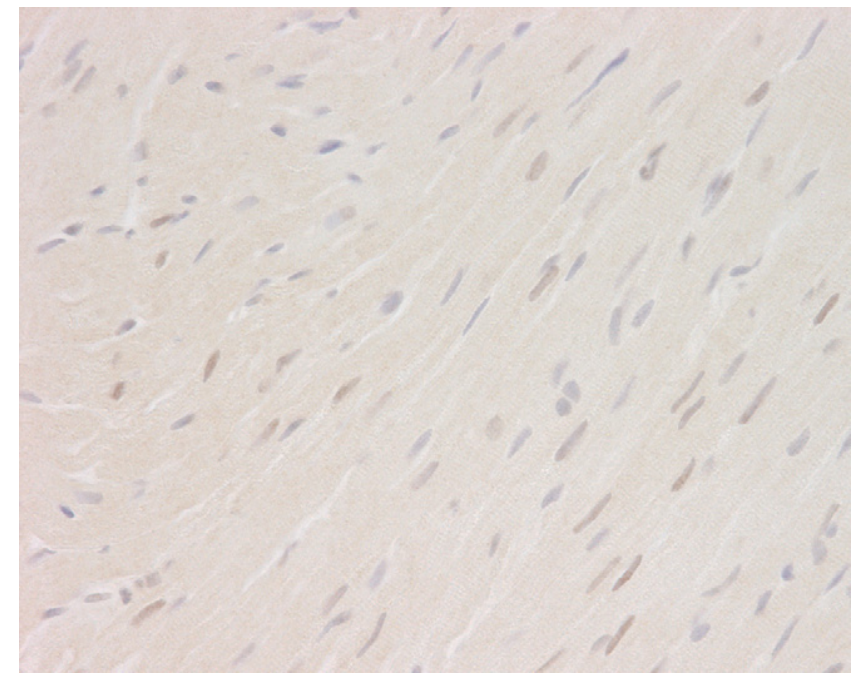

Figure E2. Section of left ventricular myocardium 21 days after intracoronary delivery of mesenchymal stem cells $(400 \times)$.

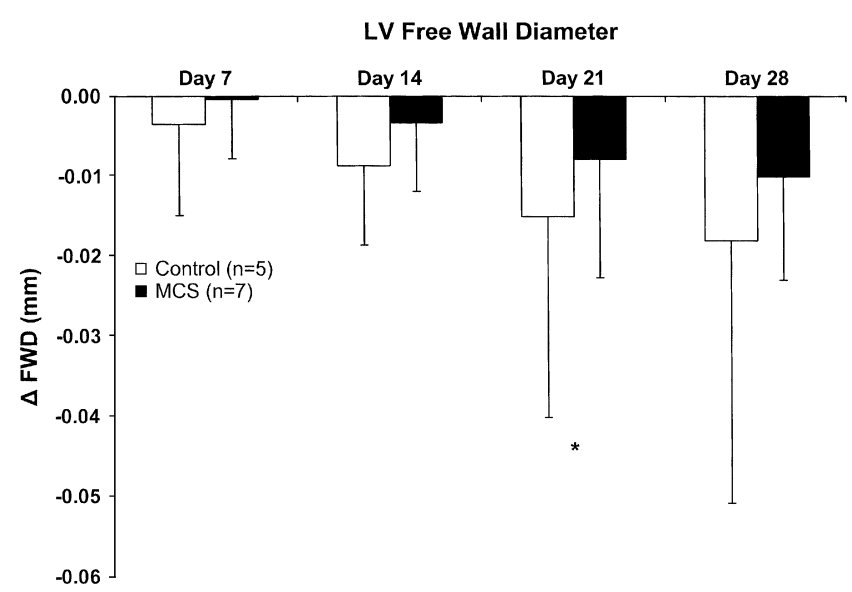

Figure E4. Changes in free wall diameter (FWD) from day 0 at each time interval. * $P<.01$ MSC versus control group. MSC, Mesenchymal stem cell. 